\title{
Inestabilidad microsatelital y cáncer gástrico
}

\author{
Microsatellite instability and gastric cancer
}

\author{
Gabriela Negrete-Tobar ${ }^{1} \mathbb{D}$, Alejandro González-Motta ${ }^{2}$ DD, Oscar Alberto Messa-Botero ${ }^{3}$ (D), \\ Juan Carlos Galvis ${ }^{4} \mathbb{D}$, Isabella Garciandía-Rozo' $\mathbb{D}$, Juan Sebastián Álvarez-Martínez $\mathbb{D}^{\mathbb{D}}$, \\ Juliana Pineda-Ortega ${ }^{1} \mathbb{D}$, Natalia Londoño de Vivero ${ }^{1} \mathbb{D}$, Ricardo Bruges-Maya ${ }^{5} \mathbb{D}$
}

1 Estudiante de medicina, Pontificia Universidad Javeriana, Bogotá, D.C., Colombia.

2 Médico, especialista en Oncología y Radioterapia, Centro Javeriano de Oncología; Hospital Universitario San Ignacio, Bogotá, D.C., Colombia.

3 Médico, especialista en Patología oncológica; profesor asistente, Facultad de Medicina, Pontificia Universidad Javeriana, Centro Javeriano de Oncología; Hospital Universitario San Ignacio, Bogotá, D.C., Colombia.

4 Médico, especialista en Oncología y Radioterapia, profesor ad honorem, Facultad de Medicina, Pontificia Universidad Javeriana, Centro Javeriano de Oncología; Hospital Universitario San Ignacio, Bogotá, D.C., Colombia.

5 Médico, especialista en Oncología clínica; profesor asistente, Facultad de Medicina, Pontificia Universidad Javeriana, Centro Javeriano de Oncología; Hospital Universitario San Ignacio, Bogotá, D.C., Colombia.

\section{Resumen}

La inestabilidad microsatelital es causada por una alteración de los sistemas de reparación de apareamiento incorrecto, que puede afectar los microsatélites dentro de todo el genoma humano, produciendo errores en su replicación. Los estudios publicados, principalmente en la literatura inglesa, han encontrado que algunos tumores, como los gástricos, pueden expresar inestabilidad microsatelital.

En la siguiente revisión de tema, se presenta una descripción de los sistemas de reparación de apareamientos incorrectos y su relación con la presencia de inestabilidad microsatelital en los tumores gástricos, así como su posible utilidad clínica, como factor asociado en la respuesta al tratamiento con inmunoterapia en los pacientes con dicha patología.

Palabras clave: cáncer; gástrico; inestabilidad microsatelital, inmunoterapia, tratamiento.

\begin{abstract}
Microsatellite instability is caused by an alteration of the mismatch repair systems, which can affect microsatellites within the entire human genome, causing errors in their replication. Published studies, mainly in the English literature, have found that some tumors, such as gastric ones, can express microsatellite instability. In this review, a description of the mismatch repair systems and their relationship with the presence of microsatellite instability in gastric tumors is presented, as well as its possible clinical utility, as an associated factor in the response to immunotherapy treatment, in patients with gastric cancer.
\end{abstract}

Keywords: cancer; gastric; microsatellite instability, immunotherapy, treatment.

Fecha de recibido: 13/05/2020 - Fecha de aceptación: 30/09/2020

Correspondencia: Alejandro González Motta, Carrera 7 \# 40 62, Hospital Universitario San Ignacio, Centro Javeriano de Oncología. Bogotá, D.C., Colombia. Teléfono: 3102602188. Correo electrónico: alejo.g.motta@gmail.com

Citar como: Negrete-Tobar G, González-Motta A, Messa-Botero OA, Galvis JC, Garciandía-Rozo I, Álvarez-Martínez JS, et al. Inestabilidad microsatelital y cáncer gástrico. Rev Colomb Cir. 2021;36:120-31. https://doi.org/10.30944/20117582.658

Este es un artículo de acceso abierto bajo una Licencia Creative Commons - BY-NC-ND https://creativecommons.org/licenses/by-nc$\mathrm{nd} / 4.0 /$ deed.es 


\section{Introducción}

El cáncer gástrico es el quinto cáncer más frecuente y la tercera causa de muerte por cáncer en el mundo ${ }^{1}$. En Colombia, ocupa el cuarto lugar en incidencia, con una tasa ajustada por edad de 12,8 por 100.000 habitantes, y el primer lugar en mortalidad, con una tasa ajustada por edad de 9,4 por 100.000 habitantes. En hombres, es el segundo más frecuente en incidencia, mientras que en las mujeres ocupa la quinta posición ${ }^{2}$. El tratamiento depende del estadio de la enfermedad, y abarca diferentes modalidades como cirugía, quimioterapia neoadyuvante, radioterapia adyuvante e inmunoterapia ${ }^{3,4}$.

La inestabilidad microsatelital (IMS) se presenta cuando hay una falla de los genes reparadores de apareamientos incorrectos. Se ha reportado que algunos tumores gástricos presentan IMS; este hallazgo en las neoplasias se considera un fenotipo "hipermutador" ${ }^{4}$ y puede representar un factor en la elección de tratamiento.

En los pacientes occidentales, se estima que la prevalencia de la IMS es del $22 \%{ }^{5}$. En los casos de cáncer gástrico esporádico, más del $50 \%$ de los casos de inestabilidad microsatelital se asocian a la metilación del gen hMLH1, convirtiéndose esta en la primera causa ${ }^{6}$. La IMS en los tumores gástricos ha sido estudiada como posible marcador de respuesta positiva a la inmunoterapia; es así como para el pembrolizumab, se encontró respuesta al tratamiento en el 57,1\% de los pacientes que expresaban la inestabilidad, en comparación con el $9 \%$ de los que no la expresaban ${ }^{7}$.

La literatura en español que habla de la inestabilidad microsatelital y su relación con el tratamiento del cáncer gástrico y de la unión gastroesofágica es limitada ${ }^{8,9}$, razón por la cual, se realiza esta revisión no sistemática de la literatura.

\section{Epidemiología}

El cáncer gástrico, corresponde a uno de los tumores con mayor incidencia y mortalidad. A nivel mundial, en 2018 se presentaron cerca de un millón de casos nuevos de cáncer de estómago (cardial y no cardial) y un estimado de 783.000 muertes, siendo la incidencia dos veces mayor en hombres que en mujeres ${ }^{1}$.

En Colombia, el cáncer de estómago es el cuarto cáncer con mayor incidencia en ambos sexos ${ }^{2}$. El patrón geográfico del riesgo de muerte por cáncer gástrico en hombres y mujeres muestra una marcada diferencia entre las regiones montañosas y las regiones costeras del territorio nacional. La región del Pacífico, y en especial la del Caribe, se relacionan con un riesgo de muerte por cáncer gástrico inferior al promedio nacional, en contraste con el patrón observado en la región montañosa, que tiene un riesgo elevado de cáncer gástrico, siguiendo el curso de las cordilleras, desde Nariño en el sur, hasta el departamento de Santander, en el nororiente ${ }^{10}$.

La supervivencia a 5 años para pacientes con cáncer gástrico en Japón está entre un 40 - 60 \% y para países desarrollados de occidente se estima en un $20 \%{ }^{3}$. Estas diferencias, son un reflejo del estadio de diagnóstico de la enfermedad. Por ejemplo, en Estados Unidos más del $65 \%$ de los pacientes con cáncer gástrico son diagnosticados en un estadio avanzado (T3 o T4) y cerca del $80 \%$ de los pacientes tienen compromiso nodal en el momento del diagnóstico ${ }^{3}$.

\section{Tratamiento del cáncer gástrico}

El tratamiento de los pacientes con cáncer gástrico es multimodal, y en él intervienen tres conductas principales: la cirugía, el tratamiento sistémico y el tratamiento con radioterapia. La cirugía representa el eje central del tratamiento con intención curativa, sin embargo, debido a la persistencia de una alta tasa de recaída local y de progresión sistémica, se requiere adicionar, en la mayoría de los pacientes, un tratamiento neoadyuvante o adyuvante. La mejor forma y secuencia para integrar estas tres conductas, según el estado de la enfermedad, es motivo de estudio permanente, especialmente en pacientes con cáncer gástrico avanzado, quienes tienen el peor pronóstico y en quienes se busca optimizar el tratamiento, con el objetivo de mejorar los desenlaces oncológicos.

Actualmente no existe un tratamiento adyuvante o neoadyuvante estándar globalmente 
aceptado, y es diferente en Estados Unidos, Europa y Asia, donde se han desarrollado sus propias estrategias de terapia adyuvante ${ }^{11}$. En los Estados Unidos, el tratamiento adyuvante con radioterapia y quimioterapia concurrente es el estándar, basado en la investigación SWOG 9008/INT0116, en la que se encontró un beneficio en la supervivencia ${ }^{12}$. En otro estudio, se aleatorizaron los pacientes posoperatorios con cáncer gástrico o de la unión esófago-gástrica, estadio Ib-IV, en dos grupos: solo observación o tratamiento neoadyuvante con quimio-radioterapia. El seguimiento a 10 años mostró que los pacientes que recibieron tratamiento con radio-quimioterapia, disminuyeron de forma significativa la probabilidad de morir en un $32 \%$, comparado con los pacientes que no la recibieron $\left(\mathrm{IC}_{95 \%} 1,10\right.$ a 1,$\left.60 ; \mathrm{p}=0,0046\right){ }^{13}$.

En Europa, la quimioterapia neoadyuvante es comúnmente utilizada de forma previa al procedimiento quirúrgico, con el objetivo de disminuir el tamaño y la extensión del tumor, evitando además procedimientos quirúrgicos de alto riesgo, al disminuir la posibilidad de aparición de metástasis a distancia ${ }^{14}$. Múltiples estudios han comparado la cirugía sola versus el tratamiento con quimioterapia neoadyuvante, demostrando un beneficio en la supervivencia de los pacientes que la recibieron ${ }^{15-18}$. Uno de estos estudios, es el estudio "MAGIC" ${ }^{16}$, en el cual se aleatorizaron los pacientes con cáncer gástrico o adenocarcinomas de la unión esófago-gástrica potencialmente curables, en un grupo al que solo se le realizó cirugía y en otro grupo que, además de la cirugía recibió quimioterapia preoperatoria y postoperatoria. A los 49 meses de seguimiento, los pacientes que recibieron quimioterapia preoperatoria y cirugía tuvieron una disminución significativa del riesgo de muerte en un $25 \%$, en comparación con los pacientes que solo fueron llevados a cirugía.

En Asia, la conducta descrita es el uso de quimioterapia adyuvante exclusiva, como fue recomendado por el metaanálisis "GASTRIC", el estudio japonés S-1 y el estudio "CLASSIC" 11. Estos estudios demostraron que la quimioterapia adyuvante para cáncer gástrico resecado, se asociaba con una reducción en el riesgo de muerte de entre un $18 \%$ y un $30 \%{ }^{11}$.

\section{Pronóstico de los pacientes con cáncer gástrico}

El pronóstico de los pacientes varía de acuerdo con la localización del tumor, la extensión de la enfermedad y la ubicación geográfica de los pacientes. Para la supervivencia a 5 años, el factor pronóstico más importante es la estadificación, siendo del $57 \%$ al $71 \%$ para el estadio I, del $33 \%$ al $47 \%$ para el estadio II, de $9 \%$ al $20 \%$ para el estadio III, y del $4 \%$ para el estadio IV. La estadificación también se relaciona con la recurrencia ${ }^{16}$. En occidente, se alcanzan supervivencias globales a 5 años del $50 \%$, incluyendo pacientes de todos los estadios, porcentaje inferior con respecto a la supervivencia en los países asiáticos, donde se alcanza una supervivencia global a 5 años del $62 \%{ }^{19}$.

\section{Sistema de reparación del ADN e inestabilidad microsatelital}

El proceso de replicación del ADN es un proceso constante que se produce de forma activa en la mayoría de las líneas celulares eucariotas y debe ser lo más preciso posible. La copia que se está produciendo, idealmente debe ser idéntica, por lo que las células replican su ADN con una alta tasa de exactitud, ocurriendo menos de una mutación por genoma durante la división celular ${ }^{20}$. Las encargadas de la replicación del ADN son la ADN polimerasa épsilon y delta, las cuales producen alrededor de un error por cada $10^{4}-10^{5}$ nucleótidos polimerizados ${ }^{21}$.

Las células eucarióticas tienen un grupo de genes que se encargan de mantener la estabilidad del ADN y de realizar la reparación del daño del mismo. Cuando se produce un error en estas vías, puede originarse el cáncer, ya que uno de los pilares centrales para su desarrollo es la inestabilidad genómica y la "hipermutabilidad". La alteración de estos procesos representa una mayor susceptibilidad para la aparición de una enfermedad neoplásica ${ }^{22,23}$.

Existen diferentes vías involucradas en la reparación de los daños específicos del ADN, entre ellas se encuentran la reparación de apareamientos incorrectos ("DNA mismatch repair" en inglés), que 
se encarga de reparar las inserciones o deleciones inapropiadas de nucleótidos, de errores de apareamiento de un solo nucleótido; la reparación por escisión de bases, la cual permite la reparación del rompimiento de una cadena y su recombinación homóloga; la reparación por escisión de nucleótidos, que repara los aductos de ADN; la reparación no homóloga o unión de extremos no homólogos, que permite reparar rompimientos de la doble cadena; la vía de la anemia de Fanconi, en la cual se reparan uniones entre la cadena, y la vía de la metil-guanina-metil-transferasa del $A D N$, que permite reparar los aductos de metil-guanina ${ }^{22,24}$.

Cuando una célula se divide, ocurren aproximadamente 100.000 errores, los cuales deben ser corregidos inicialmente por la actividad de la exonucleasa, de la polimerasa épsilon y delta. En caso de no ser arreglados, se debe realizar a través de las vías de reparación de apareamientos incorrectos, para que la copia del ADN logre ser lo más parecida posible a la cadena de ADN usada como modelo ${ }^{4,21}$. Esta vía se compone de los heterodímeros de MSH2 y MSH6, los cuales se encargan de detectar los errores de apareamiento entre base y base o 1-2 inserciones, o deleciones en asas (Insertion/deletion loop, "IDL" en inglés) de pares de bases, y de los heterodímeros formados por MSH2 y MSH3, que reconocen grandes inserciones o deleciones de las asas ${ }^{25}$. Las inserciones o deleciones en asas, se producen por errores de la polimerasa durante la replicación de las regiones del ADN que contienen repeticiones cortas en tándem, conocidas como microsatélites, las cuales son repeticiones de nucleótidos de mono o tetranucleotidos 4 . MLH1 y PMS2 forman el heterodímero de Mut L que forma un complejo ternario con uno de los complejos de Mut $\mathrm{S}$ (heterodímero resultante de la unión de MSH2 y MSH6), que se une al sitio del apareamiento incorrecto $y$, junto con la exonucleasa 1, el antígeno nuclear de proliferación celular y la ADN polimerasa delta, realizan la escisión del nucleótido erróneo y la síntesis del ADN correcto ${ }^{4}$.

\section{La inestabilidad microsatelital}

Los microsatélites son repeticiones cortas en tándem de ADN compuestas por 1 a 6 pares de bases o más, y se encuentran distribuidos a lo largo del genoma humano ${ }^{22}$. Los microsatélites están en las regiones codificantes y no codificantes del genoma, y adicionalmente tienen una susceptibilidad para desarrollar mutaciones debido a que se repiten de forma frecuente ${ }^{22}$. Cuando se producen errores en la reparación de apareamientos incorrectos, ocurren errores en la replicación de los microsatélites y, por ende, se genera la inestabilidad microsatelital, la cual puede alterar el marco de lectura, resultando en la producción de proteínas truncadas o inactivas ${ }^{26}$. Adicionalmente, las cadenas transcritas de RNA que tienen inestabilidad microsatelital pueden ser blanco de la degradación, mediada por mutaciones terminadoras ${ }^{27}$.

El término de inestabilidad microsatelital se refiere al aumento de mutaciones, cuando se presenta un error en la actividad de la reparación de apareamientos incorrectos, generando un fenotipo proclive a un incremento en el número de mutaciones, que producen cambios en las secuencias de los microsatélites ${ }^{22}$. Este aumento, se empieza a producir por cambios en las secuencias de microsatélites, causados por mutaciones de la línea germinal o silenciamiento esporádico epigenético, llevando a la sustitución, inserción o deleción de nucleótidos en las regiones de los microsatélites durante la reparación del ADN ${ }^{6,22,28}$. Los tumores con inestabilidad microsatelital tienen una alta tasa de sustituciones de nucleótidos únicos y de mutaciones, siendo considerados como tumores "hipermutados" 4

Los defectos capaces de producir inestabilidad microsatelital son los de la vía de reparación de apareamientos incorrectos, producidos por mutaciones germinales o alteraciones epigenéticas en los genes de reparación de apareamientos incorrectos, como MLH1, MSH2, MSH6 o PMS2. También pueden presentarse deleciones en los genes EPCAM, los cuales producen represión por metilación de los genes MSH2 y, por tanto, un fenotipo muy similar al encontrado en el síndrome de Lynch ${ }^{22,29}$. En los casos de cáncer gástrico esporádico, más del $50 \%$ de los casos con inestabilidad microsatelital tienen "hipermetilación" epigenética del promotor de hMLH1, el $12 \%$ - $15 \%$ por mutaciones en hMLH1 y hMSH2, y el 
resto, por inactivación de la expresión del gen por alteraciones genéticas o epigenéticas desconocidas ${ }^{6}$ (tabla 1 ).

\section{Prevalencia de inestabilidad microsatelital}

En los países occidentales, se ha estimado una prevalencia del $22 \%$ de inestabilidad microsatelital en los pacientes con cáncer gástrico ${ }^{5}$. En Colombia se han realizado dos estudios, uno de ellos en el Hospital Universitario de Santander, donde se encontró una prevalencia del $17 \%$ en pacientes con cáncer colorrectal o gástrico, utilizando el marcador BAT- $26^{8}$. El otro, es un estudio multicéntrico, que analizó el DNA genómico de cáncer gástrico y de tejido no tumoral de 22 pacientes coreanos, 20 colombianos y 26 de Estados Unidos ${ }^{9}$. En este material tumoral fueron amplificados 5 marcadores de microsatélites, encontrando que la prevalencia de la IMS en la población de pacientes colombianos fue del $15 \%$, comparada con una prevalencia del $50 \%$ en individuos coreanos ${ }^{9}$.

\section{Síndromes asociados a la inestabilidad microsatelital}

El síndrome de Lynch es una enfermedad autosómica dominante, descrita por primera vez en 1966 por Henry Lynch y colaboradores ${ }^{30}$, y posterior- mente denominada síndrome de Lynch por Boland y Troncale en $1984^{31}$. Este síndrome corresponde a la causa más común de cáncer colorrectal heredado y representa un $3 \%$ de los casos nuevos de cáncer de colon ${ }^{32}$. Este síndrome se presenta por mutaciones germinales en los genes reparadores de apareamientos incorrectos, o por la pérdida de la expresión del gen MSH2, por una deleción en el gen EPCAM, generando un aumento en el riesgo de desarrollar neoplasias malignas colorrectales ${ }^{22,32}$. Se ha planteado que para que se desarrollen neoplasias en el síndrome de Lynch, estas ocurren de acuerdo con el modelo del "doble golpe" de Knudson. En este modelo, los individuos afectados heredan un defecto de la línea germinal que los predispone a un riesgo aumentado de desarrollar cáncer, lo cual solo sucede cuando se presenta el segundo golpe, que genera la pérdida de la función del alelo normal, heredado del padre o madre no afectada por la mutación. Esto da como resultado una pérdida de la función completa de uno de los genes reparadores de apareamientos incorrectos y genera en esta célula una inestabilidad microsatelital y una predisposición para la generación del tumor $22,32,33$. La posibilidad de la aparición de estas neoplasias parece estar asociada con el sexo y con el gen que presenta la mutación ${ }^{32}$.

El riesgo de desarrollar cáncer colorrectal es de un $30 \%$ a un $74 \%$, en individuos portadores

Tabla 1. Genes implicados en la reparación del ácido desoxirribonucleico (ADN) y la inestabilidad microsatelital (IMS).

\begin{tabular}{lll}
\hline Heterodímeros & \multicolumn{1}{c}{ Función en la reparación } & \multicolumn{1}{c}{ Alteración de IMS } \\
\hline MSH2 y MSH6 & $\begin{array}{l}\text { Detectan errores de apareamiento entre ba- } \\
\text { ses o IDL en bases }\end{array}$ & $\begin{array}{l}\text { MSH2: mutación, alteración en EPCAM } \\
\text { genera metilación } \\
\text { MSH6: inactivación }\end{array}$ \\
\hline MSH2 Y MSH3 & $\begin{array}{l}\text { Detectan grandes inserciones o deleciones } \\
\text { en asas }\end{array}$ & $\begin{array}{l}\text { MSH2: mutación, alteración en EPCAM } \\
\text { genera metilación }\end{array}$ \\
\hline MLH1 Y PMS2 & $\begin{array}{l}\text { Se unen al sitio de apareamiento incorrecto } \\
\text { y con cofactores realizan la escisión de nu- } \\
\text { cleótido erróneo y síntesis de ADN correcto }\end{array}$ & $\begin{array}{l}\text { MLH1: hipermetilación epigenética de su } \\
\text { promotor y mutación. } \\
\text { PSM2: inactivación }\end{array}$ \\
\hline
\end{tabular}

IMS: inestabilidad microsatelital. ADN: ácido desoxirribonucleico. MSH2: gen codificante proteína de reparación de apareamientos incorrectos Msh2. MSH6: gen codificante de la proteína de reparación de apareamientos incorrectos Msh6. MSH3: gen codificante de la proteína de reparación de apareamientos incorrectos Msh3. MLH1: gen codificante de la proteína de reparación de apareamientos incorrectos Mlh1. PSM2: gen codificante de la proteína de reparación de apareamientos incorrectos Psm2. IDL: inserciones o deleciones en asas (insertion/deletion loop en inglés). EPCAM: gen codificante de la molécula de adhesión celular epitelial (EpCAM). 
de una mutación de MLH1 o MSH2 (32); de un $10 \%$ a un $22 \%$, en pacientes con mutaciones de MSH $6{ }^{34}$, y de un $15 \%$ a un $20 \%$ en individuos portadores de mutaciones de PMS2 ${ }^{35}$. Los pacientes con síndrome de Lynch, tienen algunas características propias de la enfermedad, entre las que se encuentran: una edad media menor en el momento de diagnóstico del cáncer colorrectal, entre 44 y 61 años comparado con 69 años en los casos de cáncer colorrectal esporádicos; la aparición predominante en el lado derecho del colon y una alta tasa de tumores metacrónicos; y una histología pobremente diferenciada, en ocasiones con células en anillo de sello y la presencia de abundante mucina por fuera de la célula ${ }^{32}$.

La secuencia de trasformación de un pólipo en adenocarcinoma es más rápida en pacientes con síndrome de Lynch, al compararla con la de los pacientes con cáncer esporádico, siendo de 35 meses en el primer caso, versus 10 - 15 años en los pacientes con cáncer esporádico ${ }^{36}$. Los pacientes con síndrome de Lynch también tienen un riesgo aumentado para desarrollar neoplasias malignas extra-colónicas, entre las que se encuentran: cáncer de endometrio, carcinoma transicional de las vías urinarias, adenocarcinomas de ovario, estómago, tracto hepatobiliar, intestino delgado, glioblastoma y neoplasias de las glándulas sebáceas de la piel ${ }^{32}$. El mayor riesgo es el de desarrollar cáncer de endometrio y se puede estimar en un $31,5 \%$ en mujeres con mutaciones en los genes de MLH1 y MSH2 ${ }^{37}$.

El síndrome de Muir-Torre es una variante rara de síndrome de Lynch, en el cual los pacientes o familias con síndrome de Lynch, desarrollan neoplasias de glándulas sebáceas y/o neoplasias de los folículos pilosos ${ }^{38}$, se puede desarrollar con mutaciones en cualquiera de los genes de reparación de apareamientos incorrectos, pero es más común en pacientes con mutaciones de $\mathrm{MSH} 2{ }^{39}$, la IMS, está presente en estos pacientes con tumores de colon o piel ${ }^{40}$. También se pueden desarrollar síndromes de deficiencia constitucional de los genes de reparación de apareamiento incorrecto, en los que los pacientes o familias afectadas presentan mutaciones bi-alélicas, que se manifiestan como mancha café con leche, y desarrollan tumores colorrectales, otros tumores asociados al síndrome de Lynch, oligopoliposis en el intestino delgado o colon, tumores cerebrales y neoplasias hematológicas ${ }^{41,42}$.

\section{Técnicas de detección de la Inestabilidad Microsatelital}

Existen varios métodos utilizados para detectar un tumor con defectos en la vía de reparación de apareamientos incorrectos es decir, con inestabilidad microsatelital. Entre estos métodos se encuentra la reacción en cadena de la polimerasa para amplificación de las secuencias de microsatélites, la detección por inmunohistoquímica (IHQ) de la expresión de proteínas de reparación de apareamientos incorrectos y la secuenciación de nueva generación para detección de inestabilidad microsatelital.

La amplificación por reacción en cadena de la polimerasa, con iniciadores específicos para repetición de microsatélites, resulta en un perfil distintivo de amplificación ${ }^{43}$. Al comparar la posición del locus del microsatélite en el tumor con el tejido normal, se puede detectar la inestabilidad microsatelital como un cambio en el electroferograma ${ }^{6}$. Para lograr una estandarización entre los diferentes laboratorios, el National Cancer Institute de Estados Unidos, en 1997 recomendó utilizar un panel con 5 marcadores: BAT25, BAT26, D2S123, D5S346 y D17S250 ${ }^{44}$. Estas regiones son amplificadas en paralelo, usando la técnica de amplificación por reacción en cadena de la polimerasa, y sus tamaños son evaluados por electroferograma. Mediante este método, se pueden determinar 3 estadios basados en los diferentes tamaños de los alelos en el tejido tumoral, comparado con el tejido normal ${ }^{6,43}$. La inestabilidad microsatelital se clasifica como alta, si se encuentran dos o más de los cinco marcadores, baja, si uno de los cinco marcadores muestra inestabilidad, y estable ( $\sin$ inestabilidad), si ninguno de los marcadores está presente $^{6}$.

La técnica de detección de inestabilidad microsatelital por inmunohistoquímica, muestra características de rendimiento, similares a las de la reacción en cadena de la polimerasa, con una tasa de concordancia superior al $90 \%{ }^{45}$. Esta prueba 
se basa en que la pérdida de expresión de una sola proteína, o de una pareja hetero dimérica del complejo de reparación de apareamientos incorrectos, sugiere la presencia de un defecto en este sistema, siendo este hallazgo una evidencia indirecta de inestabilidad microsatelital.

Hay proteínas como hMLH1 o hMSH2, que son estables sin su pareja dimérica respectiva hPMS2 y hMSH6, mientras que estos no son estables sin sus contrapartes ${ }^{46}$. Los tumores con mutaciones en los genes hMLH1 o hMSH2, usualmente muestran pérdida de su respectivo dímero, sin embargo, las mutaciones en hPMS2 y hMSH6 solo muestran la pérdida de la proteína afectada ${ }^{6,43,46}$. De esta forma, la inmunohistoquímica permite determinar, cuáles genes reparadores de apareamientos incorrectos son defectuosos, sin embargo, puede dar información errónea en los casos de mutaciones sin sentido en los genes hMLH1 o hMSH6, ya que produce proteínas con afinidad por los anticuerpos pero con ausencia de actividad enzimática. En estos casos, solamente las técnicas de reacción en cadena de la polimerasa pueden determinar cuáles proteínas son realmente funcionales ${ }^{6,44}$.

La técnica por inmunohistoquímica tiene una sensibilidad del 92,3 \% y una especificidad del 94,1 \% para la detección de inestabilidad microsatelital en el síndrome de Lynch ${ }^{47}$. Las ventajas del uso de la inmunohistoquímica son la facilidad para integrar su uso en los laboratorios de patología y su habilidad para identificar qué gen debe ser investigado para futuros análisis moleculares, en caso de que un síndrome de cáncer hereditario sea sospechado. Otra ventaja es que sólo es requerido tejido del tumor, mientras que para la reacción en cadena de la polimerasa, es necesario tejido tumoral y tejido normal ${ }^{6}$. La desventaja de la técnica de inmunohistoquímica con respecto a la amplificación por cadena de la polimerasa es que no detecta de forma directa la inestabilidad microsatelital y subestima en un $5 \%$ a $11 \%$ los tumores con inestabilidad microsatelital, debido a que en estos casos, no se observa una pérdida de las proteínas reparadoras de apareamientos incorrectos que, sin embargo, no son funcionales ${ }^{6}$.

La otra técnica para la detección de la inestabilidad microsatelital es el secuenciamiento de nueva generación, que permite realizar un secuenciamiento del ADN y de esta forma identificar las mutaciones de los genes reparadores de apareamientos incorrectos. Este método no siempre requiere el emparejamiento de muestras del tejido normal y cubre un rango más amplio de loci microsatelital, por lo que no estaría limitado como la reacción en cadena de la polimerasa a 5 loci. No obstante, los costos de este método son altos y necesita más tiempo para realizarse (incluyendo el análisis bioinformático), en comparación con los dos métodos previamente descritos ${ }^{6}$.

\section{Inmunoterapia e inestabilidad microsatelital}

Muchos estudios genómicos han mostrado que el cáncer relacionado con la deficiencia de genes reparadores de apareamientos incorrectos, junto a los tumores con defectos del dominio de exonucleasa, de la subunidad de los genes de la polimerasa épsilon (polymerase epsilon, "POLE") o polimerasa delta (polymerase delta, "POLD1"), representan en conjunto un fenotipo "hipermutador" 4 .

Se ha encontrado una asociación entre la inestabilidad microsatelital y la respuesta de tumores colorrectales al bloqueo de receptores de muerte programada ${ }^{4}$. En los tumores colorrectales con una alta tasa de inestabilidad microsatelital, se encuentra aumento en el infiltrado de linfocitos, que se correlaciona con la carga de nuevos antígenos, asociados con la producción de péptidos inmunogénicos, así como una alta expresión de diferentes puntos de control molecular, como el ligando 1 de muerte programada y el antígeno 4 asociado al linfocito T citotóxico ${ }^{22}$. Dentro de la inmunoterapia se ha considerado la inhibición del complejo PD-1/PD-L1 formado por el ligando 1 de muerte programada (PD-L1) y su receptor (PD-1), complejo que suprime la respuesta inmune mediada por células $\mathrm{T}^{13}$.

Se ha podido encontrar una sobreexpresión de PD-L1 en algunos tumores, lo que favorece el crecimiento tumoral. Para determinar la expresión de PD-L1 se utilizan múltiples métodos de medición, que pueden ser expresados por las células de respuesta inmune, es decir los macrófagos y 
linfocitos, así como por las células tumorales. Por esto, los tres métodos de medición de inmunohistoquímica se diferencian de acuerdo al tipo de células que pueden identificar ${ }^{48}$.

El primer método es la medición de la densidad de células inmunes mononucleares (mononuclear immune cell density score, "MIDS"), la cual expresa la proporción de células inmunes sobre el total de células del tumor, multiplicado por 100, y se mide en una escala de grados MIDS 0 a 4. Este método indica el grado de infiltración de células inmunitarias en el tumor y el resultado es positivo desde el grado MIDS 2, donde hay una célula inmune, por cada 100 células tumorales. El segundo método es el puntaje de proporción tumoral (tumor proportion score, "TPS"), el cual expresa la proporción de células tumorales, incluyendo a las que se tiñen parcialmente y las que lo hacen completamente, sobre el total de células tumorales, multiplicado por 100. El último método es el puntaje combinado positivo (combined positive score "CPS") que expresa el número de células teñidas sin distinción entre inmunes o tumorales, sobre el número total de células tumorales, multiplicado por 100 , que es una combinación de los dos primeros métodos. Con este método al igual que con TPS, se entregan los resultados por medio de deciles ${ }^{48}$.

La medición de PD-L1 es utilizada como un predictor de la respuesta a los medicamentos inhibidores del complejo PD-1/PD-L1 (48). Gracias al estudio de la actividad inhibitoria de los linfocitos T causada por las proteínas como PDL-1 y al CTLA-4, se han venido desarrollando múltiples anticuerpos contra los receptores o contra el antígeno, con el objetivo de producir una detención en la actividad regulatoria del sistema inmune ${ }^{22}$. La intervención en estas vías de regulación inmune, se conoce como inmunoterapia, y hasta el momento se han aprobado cinco fármacos, dirigidos al bloqueo del complejo de PD-1/PD-L1 (pembrolizumab, nivolumab, atezolizumab, durvalumab y avelumab), cada uno indicado para diferentes tipos de neoplasias y en diversas instancias de la línea de tratamiento. Para bloquear el receptor de CTLA-4, se ha desarrollado el ipilimumab, que es un anticuerpo ${ }^{49}$.
Estos medicamentos se utilizaron inicialmente en tumores con alta inmunogenicidad como el melanoma y el cáncer de pulmón de células no pequeñas, pero debido a su éxito, se ha evaluado su eficacia en otro tipo de tumores ${ }^{50}$. La FDA (Food and Drug Administration) aprobó en julio del 2017 el uso de nivolumab para el tratamiento de pacientes con tumores metastásicos de colon y recto, con alta presencia de IMS o daño en la reparación de genes reparadores de apareamientos incorrectos, que presentaban progresión a pesar del tratamiento con quimioterapia. Los resultados del estudio CheckMate-142, mostraron un control de la enfermedad en el $51 \%$ de los pacientes tras 12 semanas o más de tratamiento, y una respuesta objetiva en el 31,1 \% luego de un seguimiento de 12 meses ${ }^{22,51}$.

El mismo estudio mostró que, tras una mediana de seguimiento de 13,4 meses, el uso de nivolumab combinado con ipilimumab, logró una tasa de respuesta objetiva del $55 \%$ y un control de la enfermedad por 12 semanas o más en el $80 \%$ de los pacientes, por lo que esta combinación fue aprobada en julio de 2018 para el tratamiento de este grupo de pacientes ${ }^{22,52}$. El pembrolizumab, fue aprobado por la FDA para ser utilizado como tercera línea de tratamiento en pacientes con adenocarcinomas gástricos o de la unión esófago-gástrica en estadio avanzado o metastásico, bajo los criterios de un CPS > 1 y la presencia de progresión tumoral, después de recibir dos tratamientos sistémicos previos ${ }^{48,49}$.

En conjunto con la medición pretratamiento de la expresión de PD-L1, se mide la IMS y la deficiencia de los genes reparadores de apareamientos incorrectos, que también son considerados predictores en esta línea de inmunoterapia. Por medio de estudios de fase II, se encontró que los tumores sólidos que expresan IMS y PD-L1 en conjunto, tienen una mejor respuesta al tratamiento con inmunoterapia ${ }^{53}$. Se ha encontrado una asociación entre tumores que tienen una alta inestabilidad microsatelital (MSI-H, por sus siglas en ingles) y una expresión de PD-L1. En algunos estudios se ha observado que los tumores gástricos y de la unión esófago-gástrica que tienen MSI-H, presentan una mejor respuesta al pembrolizumab como tercera 
línea de tratamiento, aunque todavía no se cuenta con la suficiente evidencia para confirmarlo ${ }^{53}$. Adicionalmente, la MSI-H y la deficiencia de genes reparadores de apareamientos incorrectos, se han relacionado con la respuesta al nivolumab en el cáncer colorrectal, por lo que se ha abierto la posibilidad a investigación, en otros tipos de cáncer ${ }^{49}$.

Aunque la expresión de PD-L1 se correlaciona con una gran respuesta objetiva al bloqueo de puntos de chequeo, el beneficio en supervivencia se puede observar independientemente de la expresión de PD-L1 en tumores como: melanoma, carcinoma urotelial, tumores escamocelulares de cabeza y cuello, carcinoma de Merkel y carcinoma de células renales ${ }^{14}$. El uso de la medición de PDL1 como único predictor de la eficacia de la inmunoterapia, con bloqueo de los puntos de chequeo, podría estar privando a un grupo de pacientes, del beneficio de este tratamiento ${ }^{10}$.

El KEYNOTE-059, un estudio de fase 2 que evaluó pacientes con cáncer gástrico y de la unión esófago-gástrica, con recaída después de dos o más tratamientos previos con quimioterapia, en el 57,1\% de los pacientes que tenían positividad de PD-L1 (usando como criterio el puntaje combinado positivo, CPS) > 1), después de utilizar el pembrolizumab como monoterapia, se encontró una tasa de respuesta del $16 \%$ (IC95\% $11 \%$ $23 \%$ ), mientras los pacientes con tumores PD-L1 negativos tuvieron una tasa de respuesta general del $6 \%$ (IC95\% $3 \%-13 \%$ ). Siete pacientes de este estudio tenían tumores con alto grado de inestabilidad microsatelital y tuvieron una tasa de respuesta global del 57,1 \% (IC95\% 18,4 \% - 90,1\%) y una tasa de control de la enfermedad del 71,4\% (IC ${ }_{95 \%} 29 \%$-96,3 \%). En cambio, en aquellos pacientes con tumores sin alto grado de inestabilidad microsatelital, independiente del estado de PD-L1, se encontró una tasa de respuesta general del $9 \%$ (IC ${ }_{95 \%} 5,1 \%$ - 14,4 \%) y una tasa de control de la enfermedad del 22,2 \% (IC95\% 16,1 \% - 29,2 \%) ${ }^{7}$.

\section{Posible asociación de inestabilidad microsatelital y quimioterapia}

Se ha considerado que la deficiencia de genes reparadores de apareamiento incorrectos y la presencia MSI-H están asociadas con un efecto pronóstico positivo en pacientes tratados solo con cirugía, y un pronóstico negativo en pacientes que reciben adyuvancia de quimioterapia ${ }^{54}$. Esta hipótesis se basa en el hecho de que, los tumores MSI-H tienen un infiltrado inmune grande, que puede permitir que el sistema inmunitario entre en contacto con múltiples antígenos tumorales y posteriormente realice una supresión de la micrometástasis residual. En este escenario, la quimioterapia puede tener un efecto negativo mediante la producción de una supresión del sistema inmunitario, reduciendo el posible beneficio, derivado de la interacción entre el sistema inmunitario y el tumor ${ }^{54}$. Se requieren más estudios y una metodología específica para determinar si esta hipótesis es correcta.

\section{Inestabilidad microsatelital y pronóstico de cáncer gástrico}

En un metaanálisis que buscó establecer la relación entre las características clínico-patológicas y la supervivencia global en presencia de IMS, se encontró que los tumores gástricos que presentan una MSI-H se encuentran en pacientes mayores de 65 años, de sexo femenino, con localización distal del tumor, tipo histológico intestinal, ausencia de metástasis ganglionares y estadio temprano ${ }^{55}$. En este metaanálisis, los pacientes con tumores con MSI-H, mostraron un OR agrupado de 0,69 (IC ${ }_{95 \%}$ 0,56-0,86\%; $<<0,001$ ), observando una mayor supervivencia global, comparada con la de los pacientes con tumores sin IMS ${ }^{55}$.

\section{Conclusión}

La presencia de inestabilidad microsatelital en pacientes con cáncer gástrico puede ser un factor que permita tomar decisiones clínicas, dado que se ha asociado a una mejor respuesta a la inmunoterapia, y la identificación rutinaria en estos pacientes podría direccionar el tratamiento. Sin embargo, en Latinoamérica, donde el cáncer gástrico representa una importante causa de mortalidad, los datos de prevalencia de inestabilidad microsatelital en este grupo de pacientes son insuficientes. Se requieren más estudios que permitan establecer la prevalencia de la inestabilidad microsatelital en los pacientes con cáncer gástrico, 
y su impacto en la respuesta a los tratamientos con inmunoterapia.

\section{Cumplimiento de normas éticas}

Consentimiento informado: Por tratarse de un artículo de revisión, este trabajo no requiere consentimiento informado.

Conflictos de interés: ninguno declarado por los autores.

Financiación: autofinanciado por los autores.

Contribución de los autores: Concepción y diseño del estudio, análisis e interpretación de los resultados y redacción del manuscrito: Gabriela Negrete, Alejandro González, Oscar Alberto Messa, Juan Carlos Galvis.

Adquisición de datos, Análisis e interpretación de datos, redacción y revisión crítica y final del manuscrito: Isabella Garciandía, Garcianda, Juan Sebastián Álvarez, Juliana Pineda, Natalia Londoño, Ricardo Bruges, Juan Sebastián Álvarez, Juliana Pineda, Natalia Londoño, Ricardo Bruges.

\section{Referencias}

1. Bray F, Ferlay J, Soerjomataram I, Siegel RL, Torre LA, Jemal A. Global cancer statistics 2018: GLOBOCAN estimates of incidence and mortality worldwide for 36 cancers in 185 countries. CA Cancer J Clin. 2018;68:394-424. https://doi.org/10.3322/caac.21492

2. Organization WH. Globocan 2018 Lyon, France: International Agency for Research on Cancer; 2018 [cited 2019 February 24, 2019]. Available from: http://gco.iarc.fr/today/fact-sheets-populations

3. Sandler S. Esophagogastric junction and gastric adenocarcinoma: neoadjuvant and adjuvant therapy, and future directions. Oncology (Williston Park). 2014;28:505-12.

4. Nebot-Bral L, Brandao D, Verlingue L, Rouleau E, Caron O, Despras E, et al. Hypermutated tumors in the era of immunotherapy: The paradigm of personalized medicine. Eur J Cancer. 2017;84:290-303. https://doi.org/10.1016/j.ejca.2017.07.026

5. Cancer Genome Atlas Research N. Comprehensive molecular characterization of gastric adenocarcinoma. Nature. 2014;513(7517):202-9. https://doi.org/10.1038/nature13480

6. Ratti M, Lampis A, Hahne JC, Passalacqua R, Valeri N. Microsatellite instability in gastric cancer: molecular bases, clinical perspectives, and new treatment approaches. Cell Mol Life Sci. 2018;75:4151-62. https://doi.org/10.1007/s00018-018-2906-9
7. Fuchs CS, Doi T, Jang RW, Muro K, Satoh T, Machado M, et al. Safety and Efficacy of Pembrolizumab Monotherapy in Patients with Previously Treated Advanced Gastric and Gastroesophageal Junction Cancer: Phase 2 Clinical KEYNOTE-059 Trial. JAMA Oncol. 2018;4:e180013.

8. Cárdenas W, Castillo A, Vargas C, Moreno O, Insuasti J. Análisis de la inestabilidad de microsatélites mediante el marcador BAT-26 en una muestra de pacientes del Hospital Universitario de Santander con diagnóstico de cáncer gástrico o colorrectal. Colombia Médica. 2008;39(Supl.2):41-51.

9. Sepulveda AR, Santos AC, Yamaoka Y, Wu L, Gutierrez O, Kim JG, et al. Marked differences in the frequency of microsatellite instability in gastric cancer from different countries. Am J Gastroenterol. 1999;94:3034-8. https://doi.org/10.1111/j.1572-0241.1999.01453.x

10. Piñeros M, Pardo C, Gamboa O, Hernández G. Atlas de Mortalidad por Cáncer en Colombia. Bogotá: Instituto Nacional de Cancerología-Instituto Geográfico Agustín Codazzi; 2010.

11. Ashraf N, Hoffe S, Kim R. Adjuvant treatment for gastric cancer: chemotherapy versus radiation. Oncologist. 2013;18:1013-21.

https://doi.org/10.1634/theoncologist.2012-0462

12. Macdonald JS, Smalley SR, Benedetti J, Hundahl SA, Estes NC, Stemmermann GN, et al. Chemoradiotherapy after surgery compared with surgery alone for adenocarcinoma of the stomach or gastroesophageal junction. N Engl J Med. 2001;345:725-30. https://doi.org/10.1056/NEJMoa010187

13. Smalley SR, Benedetti JK, Haller DG, Hundahl SA, Estes NC, Ajani JA, et al. Updated analysis of SWOG-directed intergroup study 0116: a phase III trial of adjuvant radiochemotherapy versus observation after curative gastric cancer resection. J Clin Oncol. 2012;30:2327-33. https://doi.org/10.1200/JC0.2011.36.7136

14. Soon YY, Leong CN, Tey JC, Tham IW, Lu JJ. Postoperative chemo-radiotherapy versus chemotherapy for resected gastric cancer: a systematic review and meta-analysis. J Med Imag Radiat Oncol. 2014;58:483-96. https://doi.org/10.1111/1754-9485.12190

15. Boige V, Pignon J, Saint-Aubert B, Lasser P, Conroy T, Bouché 0 , et al. Final results of a randomized trial comparing preoperative 5-fluorouracil $(\mathrm{F}) /$ cisplatin $(\mathrm{P})$ to surgery alone in adenocarcinoma of stomach and lower esophagus (ASLE): FNLCC ACCORD07-FFCD 9703 trial. J Clin Oncol. 2007;25(18_suppl):4510.

16. Cunningham D, Allum WH, Stenning SP, Thompson JN, Van de Velde CJ, Nicolson M, et al. Perioperative chemotherapy versus surgery alone for resectable gastroesophageal cancer. N Engl J Med. 2006;355:11-20.

https://doi.org/10.1056/NEJMoa055531

17. Schuhmacher C, Gretschel S, Lordick F, Reichardt $P$, Hohenberger W, Eisenberger CF, et al. Neoadjuvant chemotherapy compared with surgery alone for locally advanced cancer of the stomach and cardia: European 
Organisation for Research and Treatment of Cancer randomized trial 40954. J Clin Oncol. 2010;28:5210-8. https://doi.org/10.1200/JC0.2009.26.6114

18. Ychou M, Boige V, Pignon JP, Conroy T, Bouche O, Lebreton $\mathrm{G}$, et al. Perioperative chemotherapy compared with surgery alone for resectable gastroesophageal adenocarcinoma: an FNCLCC and FFCD multicenter phase III trial. J Clin Oncol. 2011;29:1715-21. https://doi.org/10.1200/JC0.2010.33.0597

19. Cascinu S, Labianca R, Barone C, Santoro A, Carnaghi C, Cassano A, et al. Adjuvant treatment of high-risk, radically resected gastric cancer patients with 5-fluorouracil, leucovorin, cisplatin, and epidoxorubicin in a randomized controlled trial. J Natl Cancer Inst. 2007;99:601-7. https://doi.org/10.1093/jnci/djk131

20. Drake JW, Charlesworth B, Charlesworth D, Crow JF. Rates of spontaneous mutation. Genetics. 1998; 148:1667-86.

21. Kunkel TA. DNA replication fidelity. J Biol Chem. 2004;279:16895-8.

https://doi.org/10.1074/jbc.R400006200

22. Ruiz-Banobre J, Goel A. DNA Mismatch Repair Deficiency and Immune Checkpoint Inhibitors in Gastrointestinal Cancers. Gastroenterology. 2019;156:890-903. https://doi.org/10.1053/j.gastro.2018.11.071

23. Hanahan D, Weinberg RA. Hallmarks of cancer: the next generation. Cell. 2011;144:646-74. https://doi.org/10.1016/j.cell.2011.02.013

24. Dietlein F, Thelen L, Reinhardt HC. Cancer-specific defects in DNA repair pathways as targets for personalized therapeutic approaches. Trends Genet. 2014;30:32639. https://doi.org/10.1016/j.tig.2014.06.003

25. Genschel J, Littman SJ, Drummond JT, Modrich P. Isolation of MutSbeta from human cells and comparison of the mismatch repair specificities of MutSbeta and MutSalpha. J Biol Chem. 1998;273:19895-901. https://doi.org/10.1074/jbc.273.31.19895

26. Cortes-Ciriano I, Lee S, Park WY, Kim TM, Park PJ. A molecular portrait of microsatellite instability across multiple cancers. Nature communications. 2017;8:151-8. https://doi.org/10.1038/ncomms15180

27. Muller MF, Ibrahim AE, Arends MJ. Molecular pathological classification of colorectal cancer. Virchows Archiv. 2016;469:125-34. https://doi.org/10.1007/s00428-016-1956-3

28. Jiricny J. The multifaceted mismatch-repair system. Nat Rev Mol Cell Biol. 2006;7:335-46. https://doi.org/10.1038/nrm1907

29. Kovacs ME, Papp J, Szentirmay Z, Otto S, Olah E. Deletions removing the last exon of TACSTD1 constitute a distinct class of mutations predisposing to Lynch syndrome. Human mutation. 2009;30:197-203. https://doi.org/10.1002/humu.20942

30. Lynch HT, Shaw MW, Magnuson CW, Larsen AL, Krush AJ. Hereditary factors in cancer. Study of two large mid- western kindreds. Arch Inter Med. 1966;117:206-12. https://doi.org/10.1001/archinte.1966.03870080050009

31. Boland CR, Troncale FJ. Familial colonic cancer without antecedent polyposis. Ann Inter Med. 1984;100:700-1. https://doi.org/10.7326/0003-4819-100-5-700

32. Giardiello FM, Allen JI, Axilbund JE, Boland CR, Burke CA, Burt RW, et al. Guidelines on genetic evaluation and management of Lynch syndrome: a consensus statement by the US Multi-Society Task Force on colorectal cancer. Gastroenterology. 2014;147:502-26.

https://doi.org/10.1053/j.gastro.2014.04.001

33. Knudson AG, Jr. Hereditary cancer, oncogenes, and antioncogenes. Cancer Research. 1985;45:1437-43.

34. Hendriks YM, Wagner A, Morreau H, Menko F, Stormorken A, Quehenberger F, et al. Cancer risk in hereditary nonpolyposis colorectal cancer due to MSH6 mutations: impact on counseling and surveillance. Gastroenterology. 2004;127(1):17-25. https://doi.org/10.1053/j.gastro.2004.03.068

35. Senter L, Clendenning M, Sotamaa K, Hampel H, Green J, Potter JD, et al. The clinical phenotype of Lynch syndrome due to germ-line PMS2 mutations. Gastroenterology. 2008;135(2):419-28. https://doi.org/10.1053/j.gastro.2008.04.026

36. Edelstein DL, Axilbund J, Baxter M, Hylind LM, Romans $\mathrm{K}$, Griffin CA, et al. Rapid development of colorectal neoplasia in patients with Lynch syndrome. Clin Gastroenterol Hepatol. 2011;9:340-3. https://doi.org/10.1016/j.cgh.2010.10.033

37. Quehenberger F, Vasen HF, van Houwelingen HC. Risk of colorectal and endometrial cancer for carriers of mutations of the hMLH1 and hMSH2 gene: correction for ascertainment. J Med Genet. 2005;42:491-6. https://doi.org/10.1136/jmg.2004.024299

38. Lynch HT, Lynch PM, Pester J, Fusaro RM. The cancer family syndrome. Rare cutaneous phenotypic linkage of Torre's syndrome. Arch Inter Med. 1981;141:607-11. https://doi.org/10.1001/archinte.1981.0034005 0059016

39. Kastrinos F, Stoffel EM, Balmana J, Steyerberg EW, Mercado R, Syngal S. Phenotype comparison of MLH1 and MSH2 mutation carriers in a cohort of 1,914 individuals undergoing clinical genetic testing in the United States. Cancer Epidemiol Biomarkers Prev. 2008;17:2044-51. https://doi.org/10.1158/1055-9965.EPI-08-0301

40. Entius MM, Keller JJ, Drillenburg P, Kuypers KC, Giardiello FM, Offerhaus GJ. Microsatellite instability and expression of hMLH- 1 and hMSH-2 in sebaceous gland carcinomas as markers for Muir-Torre syndrome. Clinical Cancer. 2000;6:1784-9.

41. Durno CA, Holter S, Sherman PM, Gallinger S. The gastrointestinal phenotype of germline biallelic mismatch repair gene mutations. Am J Gastroenterol. 2010;105:2449-56. https://doi.org/10.1038/ajg.2010.215 
42. Trimbath JD, Petersen GM, Erdman SH, Ferre M, Luce MC, Giardiello FM. Cafe-au-lait spots and early onset colorectal neoplasia: a variant of HNPCC? Fam Cancer. 2001;1:101-5.

43. Berg KD, Glaser CL, Thompson RE, Hamilton SR, Griffin CA, Eshleman JR. Detection of microsatellite instability by fluorescence multiplex polymerase chain reaction. J Mol Diagn. 2000;2(1):20-8. https://doi.org/10.1016/S1525-1578(10)60611-3

44. Murphy KM, Zhang S, Geiger T, Hafez MJ, Bacher J, Berg $\mathrm{KD}$, et al. Comparison of the microsatellite instability analysis system and the Bethesda panel for the determination of microsatellite instability in colorectal cancers. J Mol Diagn. 2006;8(3):305-11. https://doi.org/10.2353/jmoldx.2006.050092

45. Funkhouser WK, Jr., Lubin IM, Monzon FA, Zehnbauer BA, Evans JP, Ogino S, et al. Relevance, pathogenesis, and testing algorithm for mismatch repair-defective colorectal carcinomas: a report of the association for molecular pathology. J Mol Diagn. 2012;14(2):91-103. https://doi.org/10.1016/j.jmoldx.2011.11.001

46. Leite M, Corso G, Sousa S, Milanezi F, Afonso LP, Henrique $\mathrm{R}$, et al. MSI phenotype and MMR alterations in familial and sporadic gastric cancer. Int J Cancer. 2011;128:1606-13. https://doi.org/10.1002/ijc.25495

47. Vaccaro CA, Carrozzo JE, Mocetti E, Berho M, Valdemoros $\mathrm{P}$, Mullen E, et al. Expresión inmunohistoquímica e inestabilidad microsatelital en el síndrome de Lynch. Medicina (Buenos Aires). 2007;67:274-8.

48. Kulangara K, Zhang N, Corigliano E, Guerrero L, Waldroup S, Jaiswal D, et al. Clinical Utility of the Combined Positive Score for Programmed Death Ligand-1 Expression and the Approval of Pembrolizumab for Treatment of Gastric Cancer. Arch Pathol Lab Med. 2019;143:330-7. https://doi.org/10.5858/arpa.2018-0043-OA

49. Chen Q, Li T, Yue W. Drug response to PD-1/PD-L1 blockade: based on biomarkers. OncoTargets Ther. 2018;11:4673-83. https://doi.org/10.2147/OTT.S168313
50. Baumeister SH, Freeman GJ, Dranoff G, Sharpe AH. Coinhibitory Pathways in Immunotherapy for Cancer. Annual review of immunology. 2016;34:539-73. https://doi.org/10.1146/annurev-immunol-032414112049

51. Overman MJ, McDermott R, Leach JL, Lonardi S, Lenz HJ, Morse MA, et al. Nivolumab in patients with metastatic DNA mismatch repair-deficient or microsatellite instability-high colorectal cancer (CheckMate 142): an open-label, multicentre, phase 2 study. The Lancet Oncology. 2017;18(9):1182-91. https://doi.org/10.1016/S1470-2045(17)30422-9

52. Overman MJ, Lonardi S, Wong KYM, Lenz HJ, Gelsomino F, Aglietta M, et al. Durable Clinical Benefit With Nivolumab Plus Ipilimumab in DNA Mismatch Repair-Deficient/Microsatellite Instability-High Metastatic Colorectal Cancer. J Clin Oncol. 2018;36:773-9. https://doi.org/10.1200/JC0.2017.76.9901

53. Lin EM, Gong J, Klempner SJ, Chao J. Advances in immuno-oncology biomarkers for gqstroesophageal cancer: Programmed death ligand 1, microsatellite instability, and beyond. World J Gastroenterol. 2018;24:2686-97. https://doi.org/10.3748/wjg.v24.i25.2686

54. Smyth EC, Wotherspoon A, Peckitt C, Gonzalez D, Hulkki-Wilson S, Eltahir Z, et al. Mismatch Repair Deficiency, Microsatellite Instability, and Survival: An Exploratory Analysis of the Medical Research Council Adjuvant Gastric Infusional Chemotherapy (MAGIC) Trial. JAMA Oncol. 2017;3:1197-203.

https://doi.org/10.1001/jamaoncol.2016.6762

55. Polom K, Marano L, Marrelli D, De Luca R, Roviello G, Savelli V, et al. Meta-analysis of microsatellite instability in relation to clinicopathological characteristics and overall survival in gastric cancer. Br J Surg. 2018;105:159-67. https://doi.org/10.1002/bjs.10663 